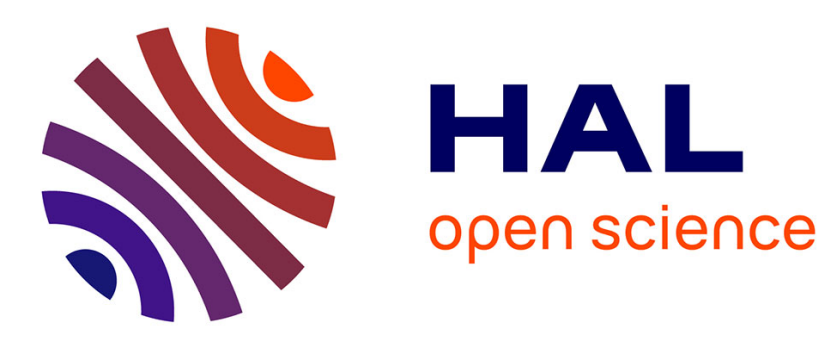

\title{
An algebraic receiver for full response CPM demodulation
}

\author{
Aline Neves, Mamadou Mboup, Michel Fliess
}

\section{To cite this version:}

Aline Neves, Mamadou Mboup, Michel Fliess. An algebraic receiver for full response CPM demodulation. International Telecommunications Symposium (ITS 2006), 2006, Fortaleza-CE, Brésil. inria00086115

\section{HAL Id: inria-00086115 https://hal.inria.fr/inria-00086115}

Submitted on 18 Jul 2006

HAL is a multi-disciplinary open access archive for the deposit and dissemination of scientific research documents, whether they are published or not. The documents may come from teaching and research institutions in France or abroad, or from public or private research centers.
L'archive ouverte pluridisciplinaire HAL, est destinée au dépôt et à la diffusion de documents scientifiques de niveau recherche, publiés ou non, émanant des établissements d'enseignement et de recherche français ou étrangers, des laboratoires publics ou privés. 


\title{
An Algebraic Receiver for Full Response CPM Demodulation
}

\author{
Aline Neves*, Mamadou Mboup** and Michel Fliess***
}

\begin{abstract}
This paper proposes a new algebraic demodulation method for full response CPM signals in AWGN channel. The method is based in a new estimation/identification theory that has also already been used for channel identification and signal deconvolution. The signals and noise are considered to be deterministic and we find an explicit formula for the recovery of the transmitted symbols. Based on this formula, the symbols are recovered blindly, needing only the knowledge of the modulation index. The method is not only simple and robust to noise, but also very fast, what enables its implementation on-line. It can also be equally used for coherent or non-coherent demodulations. The method is applied to the CPFSK and LRC families of signals showing good results.
\end{abstract}

Index Terms-CPM blind demodulation, algebraic methods, continuous-time signal, deterministic signals

\section{INTRODUCTION}

The Continuous Phase Modulation (CPM) is attractive for wireless communication systems due to its constant envelope and spectral efficiency. Despite its attractive features [1], the widespread use of CPM has been so far hindered by a high level of complexity of the demodulation process. The optimum receiver for AWGN (Additive White Gaussian Noise) channel consists of a correlator followed by a maximum-likelihood estimator using a Viterbi-like algorithm. Its complexity grows exponentially with the symbols alphabet and the modulation memory [2].

Alternatively, a number of sub-optimum detectors have been proposed, but the required computational burden is still high [3], [4]. In terms of blind methods, [5] showed that the constant modulus criterion is not capable of equalizing a CPM signal. Other methods proposed in [6], [7] present many local minima and are especially complex, using Markov chains [6] or depending on sequences where the elements are raised to the power of non-integer values [7]. In general, the proposed approaches in the literature share a common probabilistic setting in which the prior knowledge of the symbol data distribution, as well as that of the noise disturbance, is a key assumption.

On the other hand, deterministic approaches, which are usually rightly discarded because of their sensitivity to noise perturbations, may be very efficient when casted in an appropriate theoretical framework. The algebraic method proposed

*Signal Processing Laboratory for Communications (DSPCom) UNICAMP, Campinas, Brasil, CP 6101, CEP 13083-970, aline@decom.fee.unicamp.br

**UFR Math-Info, Université René Descartes \& INRIA Futurs, 45 rue des Saints-Pères 75270 Paris Cedex 06, France, mboup@math-info.univ-paris5.fr *** Laboratoire LIX \& INRIA Futurs, Ecole Polytechnique, 91128 Palaiseau, France, michel.fliess@stix.polytechnique.fr in [8] for full response CPFSK modulation, is a good example. As opposed to other deterministic approaches, the estimation technique does not rely on any optimization procedure. The paper shows how the algebraic demodulation is based on a very fast and blind on-line identification of the informationbearing symbols directly from the continuous-time received signal. Moreover, the method is robust to a wide variety of additive noise. More details about the theoretical framework can be found in [9].

In this paper we propose an algebraic demodulation method simpler and more robust then the one proposed in [8]. The method also does not rely on optimization procedures, resulting in a closed formula for the symbols recovery. This is an important characteristic especially when treating with blind techniques, since it means that the method does not present local minima. We will treat the full response modulations in AWGN channel, considering in more details the CPFSK and the LRC families of CPM signals. Even though we are going to treat here only these two cases, it is important to note that the method can also be used with other CPM families.

We start this paper explaining the problem and reviewing the CPM modulation in section II. Section III resumes what was done in [8] and section IV explains the new proposed method. Section V shows the simulation results, comparing both of the methods described earlier and section VI concludes this paper.

\section{The Problem Statement}

The CPM baseband signal is given by:

$$
u_{b}(t)=\mathcal{E} e^{j \phi(t, \boldsymbol{a})}
$$

where $\mathcal{E}$ is the signal energy and the phase is given by

$$
\phi(t, \boldsymbol{a})=2 \pi h \sum_{k=-\infty}^{\infty} a_{k} q(t-k T)
$$

The information bearing symbols $\left\{a_{k}\right\}$ are thus contained in the phase $\phi(t, \boldsymbol{a})$ where

$$
q(t)=\int_{-\infty}^{t} g(\tau) d \tau .
$$

Normally, the function $g(t)$ is a smooth pulse shape over a finite time interval $0 \leq t \leq L T$ and zero outside. Thus, $L$ is the pulse length and $T$ is the symbol period. The $M$-ary data symbols $a_{k}$ take on values $\pm 1, \pm 3, \ldots, \pm(M-1)$ and $h$ is the modulation index.

From the definition of the above class of constant amplitude modulation schemes the pulse $g(t)$ is defined in instantaneous 
frequency and its integral $q(t)$ is the phase response [1]. We normalized the pulse $g(t)$ such that

$$
\int_{-\infty}^{\infty} g(\tau) d \tau=\frac{1}{2}
$$

This restrains the maximum phase change over any symbol to $\pi h(M-1)$ radians.

Thus, by choosing different pulses $g(t)$ and varying $h$ and $M$ a great variety of CPM schemes can be obtained [1]. In this paper we are going to use a rectangular pulse of length $L$ symbol intervals, which results in the LREC modulation also known as CPFSK (Continuous Phase Frequency Shift Keying) and a raised cosine pulse also of length $L$, named LRC modulation. This results, respectively, in the following functions $q(t)$ :

$$
\begin{aligned}
q_{L R E C}(t) & =\left\{\begin{array}{ccc}
0 & t<0 \\
\frac{t}{2 L T} & 0 \leq t<L T \\
\frac{1}{2} & t \geq L T
\end{array}\right. \\
q_{L R C}(t) & =\left\{\begin{array}{cc}
0 & t<0 \\
\frac{1}{2}\left(\frac{t}{L T}-\frac{1}{2 \pi} \sin \frac{2 \pi t}{L T}\right) & 0 \leq t<L T \\
\frac{1}{2} & t \geq L T
\end{array}\right.
\end{aligned}
$$

It is important to observe that the phase continuity implies in a memory. This memory also increases with the value of $L$. The case in which $L=1$ is called full response modulation while $L>1$ is a partial response modulation. In this paper we are only going to study the first case.

Finally, for the LREC and LRC modulations respectively, the transmitted signal in the interval $n T \leq t \leq(n+1) T$ can be written as :

$$
\begin{aligned}
u(t) & =\mathcal{E} \cos \left(\omega_{c} t+\pi h \sum_{k=n-L+1}^{n} a_{k} \frac{t-k T}{L T}+\pi h \sum_{k=0}^{n-L} a_{k}\right) \\
u(t) & =\mathcal{E} \cos \left(\omega_{c} t+\pi h \sum_{k=n-L+1}^{n} a_{k}(t-k T) /(L T)\right. \\
& \left.-\frac{h}{2} \sum_{k=n-L+1}^{n} a_{k} \sin \left(\frac{2 \pi}{L T}(t-k T)\right)+\pi h \sum_{k=0}^{n-L} a_{k}\right)
\end{aligned}
$$

where $\omega_{c}=2 \pi f_{c}$, with $f_{c}$ being the carrier frequency.

In the following, we are always going to consider the signals on the time interval $n T \leq t \leq(n+1) T$, since we are interested in obtaining the symbol $a_{n}$.

\section{Algebraic Method Proposed in [8]}

The work done in [8] shows how to demodulate a full response CPFSK signal in the case of AWGN channel using an algebraic method based on a new identification/estimation theory firstly proposed in [9], [10]. This theory, which is based on differential fields, ring theory, and operational calculus, leads to the following facts:

- No precise statistical knowledge of the noise is required

- The signals are considered directly in continuous-time
- There is no distinction between stationary and nonstationary signals.

- The computations of the estimates can be done on-line.

Applying this theory to the demodulation of CPM signals, we start by defining a new variable $\tau$ such as $t=(n+\tau) T$ which enables us to rewrite (3) as

$$
\begin{aligned}
u_{n}(\tau) & =u((n+\tau) T) \\
& =\mathcal{E} \cos \left(\omega_{n} \tau+\varphi_{n}\right), \quad 0 \leq \tau<1
\end{aligned}
$$

where, considering $L=1, \omega_{n}=\omega_{c} T+\pi h a_{n}$ and $\varphi_{n}=$ $\omega_{c} n T+\pi h \sum_{k=-\infty}^{n-1} a_{k}$.

The proposed method consists of using operational calculus or, equivalently, obtaining the Laplace transform of (5), and solving a system of equations generated by the successive derivation of this equation with respect to the variable $s$. We firstly consider the noiseless case. The Laplace transform of (5) gives:

$$
\hat{x}_{n}(s)=\hat{u}_{n}(s)=\frac{s \mathcal{E} \cos \left(\varphi_{n}\right)-\omega_{n} \mathcal{E} \sin \left(\varphi_{n}\right)}{s^{2}+\omega_{n}^{2}}
$$

We can rewrite this equation as

$$
\alpha_{n} \hat{u}_{n}(s)+\beta_{n} s+\gamma_{n}=s^{2} \hat{u}_{n}(s)
$$

where we have set $\alpha_{n}=-\omega_{n}^{2}, \beta_{n}=\mathcal{E} \cos \left(\varphi_{n}\right)$ and $\gamma_{n}=$ $-\omega_{n} \mathcal{E} \sin \left(\varphi_{n}\right)$. Taking the derivative, with respect to $s$, of both sides of (7) up to order 2 yields a system of equations which enables the identification of the desired parameters and, indirectly, that of the transmitted symbol which can be obtained from $\alpha_{n}$.

$$
\begin{aligned}
\alpha_{n} \hat{u}_{n}+s \beta_{n}+\gamma & =s^{2} \hat{u}_{n} \\
\alpha_{n} \hat{u}_{n}^{\prime}+\beta_{n} & =2 s \hat{u}_{n}+s^{2} \hat{u}_{n}^{\prime} \\
\alpha_{n} \hat{u}_{n}^{\prime} & =2 \hat{u}_{n}+4 s \hat{u}_{n}^{\prime}+s^{2} \hat{u}_{n}^{\prime}
\end{aligned}
$$

Since the Laplace transform of $u_{n}(\tau)$ is not known, the terms from (8) that depend on this signal are going to be calculated returning to the time domain. Due to the fact that the multiplication by the variable $s$ in the transformed domain represents, in the time domain, a derivation with respect to time, and the derivation is not a numerically robust operation, we can divide all the terms in this system by $s^{\nu}$ where $\nu$ is a constant larger than the largest power of $s$ appearing in the system. Observing (8), we see that $\nu$ must be greater than two. Doing so, we will only have integral operators in the time domain. The system of equations will then read as:

$$
\begin{aligned}
\alpha_{n} \frac{\hat{u}_{n}}{s^{\nu}}+\frac{\beta_{n}}{s^{\nu-1}}+\frac{\gamma}{s^{\nu}} & =\frac{\hat{u}_{n}}{s^{\nu-2}} \\
\alpha_{n} \frac{\hat{u}_{n}^{\prime}}{s^{\nu}}+\frac{\beta_{n}}{s^{\nu}} & =2 \frac{\hat{u}_{n}}{s^{\nu-1}}+\frac{\hat{u}_{n}^{\prime}}{s^{\nu-2}} \\
\alpha_{n} \frac{\hat{u}_{n}^{\prime}}{s^{\nu}} & =2 \frac{\hat{u}_{n}}{s^{\nu}}+4 \frac{\hat{u}_{n}^{\prime}}{s^{\nu-1}}+\frac{\hat{u}_{n}^{\prime}}{s^{\nu-2}}
\end{aligned}
$$

Returning to the time domain, the system will be given by:

$$
\mathcal{P}_{n}\left[\begin{array}{l}
\alpha_{n} \\
\beta_{n} \\
\gamma_{n}
\end{array}\right]=\mathcal{Q}_{n}
$$


where the entries of the $3 \times 3$ and $3 \times 1$ matrices $\mathcal{P}_{n}$ and $\mathcal{Q}_{n}$ are given by iterated integrals:

$$
\begin{aligned}
\mathcal{L}^{-1}\left\{\frac{\hat{u}_{n}^{(i)}}{s^{k}}\right\} & =\int_{0}^{\lambda} \int_{0}^{\lambda_{k-1}} \cdots \int_{0}^{\lambda_{1}} r^{i} u_{n}(r) d \lambda_{k-1} \cdots d \lambda d r \\
& =\frac{1}{(k-1) !} \int_{0}^{\lambda}(\lambda-r)^{k-1} r^{i} u_{n}(r) d r
\end{aligned}
$$

The time interval $\lambda$ needs only to be sufficient for the integral to converge. This explains why the estimates of the unknown parameters can be obtained within a very short period of time. Values lower than or equal to one are sufficient. Considering $\lambda=1$ is equivalent to integrating on an interval of length $T$, i.e. of one symbol period. The averaging provided by the iterated integrals explains the robustness of the method to zero mean additive noise and especially to high frequency noise.

Identifying $\alpha_{n}$, the symbols can be recovered doing:

$$
a_{n}=\frac{\sqrt{\alpha_{n}}-\omega_{c} T}{\pi h}
$$

It is also interesting to note that the proposed method is blind, although certain modulation parameters as the carrier frequency $\omega_{c}$, the symbol period $T$ and the modulation index $h$ must be known.

\section{A. The Noise Effect}

We can consider two types of noise: a constant bias perturbation or an unstructured perturbation. In this second case, we can always write the noise as being a mean value added to a zero-mean perturbation. As mentioned before, the effect of this zero-mean perturbation at the receiver output is going to be reduced by the filtering realized by the iterated integrals. However, we still have to deal with the constant bias perturbation or the noises mean value, $b_{0}$. In this case, after (6), the received signal will be given by:

$$
\begin{aligned}
\hat{x}_{n}(s) & =\hat{u}_{n}(s)+\frac{b_{0}}{s} \\
& =\frac{s \mathcal{E} \cos \left(\varphi_{n}\right)-\omega_{n} \mathcal{E} \sin \left(\varphi_{n}\right)}{s^{2}+\omega_{n}^{2}}+\frac{b_{0}}{s}
\end{aligned}
$$

Multiplying (13) by the variable $s$ and taking the derivative also with respect to $s$, we are able to eliminate the unknown constant $b_{0}$. The resulting equation will be:

$$
\begin{aligned}
s^{5} \hat{x}_{n}^{\prime}+s^{4} \hat{x}_{n}= & s^{2} \gamma_{n}-2 s \beta_{n} \alpha_{n}+\gamma_{n} \alpha_{n} \\
& +2 \alpha_{n}\left(s^{2} \hat{x}_{n}-s^{3} \hat{x}_{n}^{\prime}\right)+\alpha_{n}^{2}\left(x+s \hat{x}_{n}^{\prime}\right)
\end{aligned}
$$

where $\alpha_{n}, \beta_{n}$ and $\gamma_{n}$ were defined in (7).

The procedure to obtain the unknown coefficients follow the same lines described previously: taking the derivative of (14) with respect to $s$, dividing by $s^{\nu}$ (with $\nu>5$ ) and returning to the time domain to obtain the iterated integrals of $x_{n}(\tau)$. Note that the fifth-order derivative of (14) will give an equation depending only on the parameter $\alpha_{n}$, equivalently to the last equation in (9).

Note that this is not the only existing procedure to eliminate $b_{0}$ in (13). For example, the multiplication of this equation by $s\left(s^{2}+\omega_{n}^{2}\right)$ followed by a third-order derivation with respect to $s$ would also eliminate $b_{0}$. The resulting parameters estimator, however, will be different. Due to the fact that the noise is generally considered to be zero-mean, white and gaussian, we will not develop the analysis of these estimators here. A detailed discussion on this subject can be found in [11].

\section{New Algebraic Method}

Considering that $\omega_{c}, T$ and $h$ are known, and that we are only interested in identifying the transmitted symbols, the method described in section III may present certain disadvantages. Firstly, we can not identify the symbols directly. As shown by (12), this identification depends on the value of $\alpha_{n}$, or, in other words, on that of $\omega_{n}^{2}$. Moreover, $\omega_{n}$ is given by $\omega_{c} T+\pi h a_{n}$. Considering the transmission at a high rate, the term $\omega_{c} T$ tends to be much higher than $\pi h a_{n}$, which means that the value of $\omega_{n}^{2}$ is basically going to vary around the value of $\left(\omega_{c} T\right)^{2}$. This makes the control of potencial errors in the identification of $a_{n}$ very difficult.

For this reason, we propose here a new algebraic method that enables the identification of the symbols directly. This new method is even simpler and more robust to noise then the previous one, as will be shown. Firstly, figure 1 shows the receiver scheme used, recovering the baseband signal from the bandpass received one.

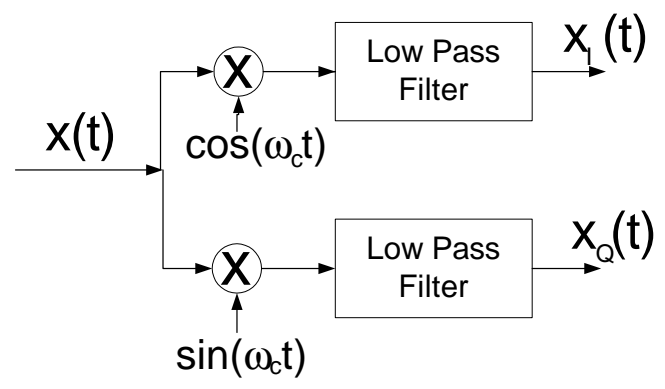

Fig. 1. Receiver scheme

\section{A. The CPFSK Modulation}

Considering the full-response ( $L=1)$ CPFSK modulation, using (3), the signals at the receiver output (figure 1) will be:

$$
\begin{aligned}
x_{I}(t) & =\frac{\mathcal{E}}{2} \cos \left(-\pi h a_{n} \frac{t-n T}{T}-\pi h \sum_{k=0}^{n-1} a_{k}\right) \\
x_{Q}(t) & =\frac{\mathcal{E}}{2} \sin \left(-\pi h a_{n} \frac{t-n T}{T}-\pi h \sum_{k=0}^{n-1} a_{k}\right)
\end{aligned}
$$

obtained by multiplying the received signal by a cosinus and sinus function at the carrier frequency, and filtering the result by a low-pass filter.

The equations (15) enables us to establish the following relation between $x_{I}$ and $x_{Q}$ :

$$
\begin{aligned}
\frac{d x_{I}(\tau)}{d \tau} & =\pi h a_{n} \frac{\mathcal{E}}{2} \sin \left(-\pi h a_{n} \tau-\pi h \sum_{k=0}^{n-1} a_{k}\right) \\
& =\pi h a_{n} x_{Q}(\tau)
\end{aligned}
$$


where we also used $t=(n+\tau) T$.

The relation is then very simple, depending only on a derivative of first order. To solve (16) and recover $a_{n}$, we can follow what was done in [8]. The Laplace transform of (16) gives us:

$$
s \hat{x}_{I}(s)-x_{I}(0)=\pi h a_{n} \hat{x}_{Q}(s)
$$

where $x_{I}(0)$ is the initial condition of $x_{I}$ in the interval $n T \leq$ $t<(n+1) T$. As $x_{I}(0)$ is unknown, it can be considered a constant bias perturbation. Taking the derivative of (17) with respect to $s$, eliminates de dependence of this constant [8]. Moreover, we need to divide all terms of (17) by $s^{\nu}$ with $\nu>1$ to have integral operators in the time domain. The symbols can then be recovered solving the following equation:

$$
a_{n}=\left(\frac{\left(s \hat{x}_{I}(s)\right)^{\prime}}{s^{\nu}}\right) /\left(\frac{\pi h \hat{x}_{Q}^{\prime}(s)}{s^{\nu}}\right)
$$

The equation (18) is going to be solved in the time domain. The terms in the right hand side of this equation are given, in the time domain, by iterated integrals of the signals $x_{I}$ and $x_{Q}$ as shown in (11). Again, the averaging provided by these integrals reduces the effect of zero mean additive noise and high frequency noise at the output estimated symbol. In the case of a constant bias perturbation, the same procedure showed in section III-A can be applied.

Remembering that we are treating sinusoids, an equation equivalent to (16) can be found taking the derivative of $x_{Q}$ instead of $x_{I}$. The resulting relation will then read as

$$
\left(s \hat{x}_{Q}\right)^{\prime}=-\pi h a_{n} \hat{x}_{I}^{\prime}
$$

A third possibility would be to add both of these relations and identify the symbol $a_{n}$ doing:

$$
a_{n}=\frac{\left(s \hat{x}_{I}\right)^{\prime}+\left(s \hat{x}_{Q}\right)^{\prime}}{\pi h\left(\hat{x}_{Q}^{\prime}-\hat{x}_{I}^{\prime}\right)}
$$

We can use the three estimations of $a_{n}$ obtained by using (17), (19) and (20) to render the result of the symbol identification more robust.

Note that this method, as the one proposed by [8], is blind and needs the knowledge of the modulation index $h$ to directly identify the desired symbol.

In addition, it can be equally used for coherent and noncoherent demodulations. This last case can be viewed as, instead of multiplying the received signal by sinusoid functions as shown in figure 1 , we substitute these functions by $\cos \left(\omega_{c} t+\theta\right)$ and a $\sin \left(\omega_{c} t+\theta\right)$ where $\theta$ is a random phase. In this case, the equations given by (17), (19) and (20) will not change. The phase $\theta$ will influence $x_{I}(t)$ and $x_{Q}(t)$ equivalently being transparent to the recovery of the symbols.

\section{B. The LRC Modulation}

The same technique can be applied to the LRC modulation. In this case, considering the full-response modulation and using (4), the signals at the output of the receiver shown in figure 1 will be:

$$
\begin{aligned}
& x_{I}(\tau)=\frac{\mathcal{E}}{2} \cos \left(-\pi h a_{n} \tau+h \frac{a_{n}}{2} \sin 2 \pi \tau-\pi a_{n} \sum_{k=0}^{n-1} a_{k}\right) \\
& x_{Q}(\tau)=\frac{\mathcal{E}}{2} \sin \left(-\pi h a_{n} \tau+h \frac{a_{n}}{2} \sin 2 \pi \tau-\pi h \sum_{k=0}^{n-1} a_{k}\right)
\end{aligned}
$$

Equations (21) lead to the following relation between $x_{I}$ and $x_{Q}$ :

$$
\begin{aligned}
\dot{x}_{I}(\tau) & =\pi h a_{n}(1-\cos (2 \pi \tau)) x_{Q}(\tau) \\
& =\pi h a_{n} \check{x}_{Q}(\tau)
\end{aligned}
$$

where $\check{x}_{Q}=(1-\cos (2 \pi \tau)) x_{Q}$.

Comparing (16) and (22), we can see that both relations are very similar. Thus, the procedure applied in section IV-A can be used here exactly in the same way, that is, the symbol estimation is obtained by calculating the Laplace transform of (22), taking the derivative with respect to the variable $s$ to eliminate the initial condition, dividing it by $s^{\nu}$ to obtain proper operators and finally, returning to the time-domain, calculating the iterated integrals.

Consequently, again as in section IV-A, it is possible to obtain three different symbol estimations: the one given by solving (22), obtaining the derivative of $x_{Q}(t)$ instead of that of $x_{I}(t)$

$$
\left(s \hat{x}_{Q}\right)^{\prime}=-\pi h a_{n} \hat{\check{x}}_{I}^{\prime},
$$

where $\check{x}_{I}=(1-\cos (2 \pi \tau)) x_{I}$ and using both relations together

$$
a_{n}=\frac{\left(s \hat{x}_{I}\right)^{\prime}+\left(s \hat{x}_{Q}\right)^{\prime}}{\pi h\left(\hat{\check{x}}_{Q}^{\prime}-\hat{\check{x}}_{I}^{\prime}\right)} .
$$

This procedure will render the identification more robust. In addition, as for the CPFSK modulation, the method is transparent to coherent or non-coherent demodulation, being able to be used without changes in both cases. Finally, the expected performance of the method for both modulations are similar, since their equations have the same structure.

Even though we will not treat the partial response case in this paper, it is interesting to notice that, in this case, we will certainly have a dependence from previous transmitted symbols since $\omega_{n}$ will depend on $L$ symbols. We will then have to be more careful in its demodulation and use previously decided symbols to decide the actual one.

\section{Simulation Results}

The simulation results shown in [8] do not include curves of bit error rate (BER). To be able to compare this method with the one proposed in this paper, figure 2 shows the BER curve for the full response CPFSK modulation obtained using (12). The modulation parameters used were: $\mathcal{E}=1.437, h=$ $0.725, T=6.25 \times 10^{-5}, \omega_{c}=60 / T, \nu=18, M=4$ and $M=8$. The interval of integration used was $\lambda=1$, which means that the integrals were evaluated numerically during the interval of one symbol period, what confirms the rapidity of the 
method. The noise was considered to be a zero-mean, white, gaussian noise. In order to evaluate the BER, $10^{5}$ symbols were transmitted.

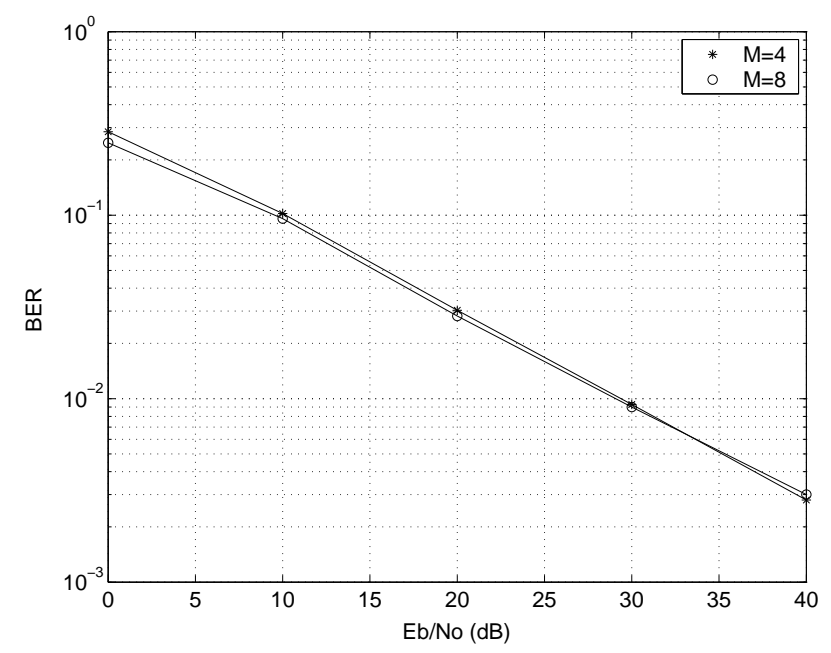

Fig. 2. Method proposed in [8]

Figure 3 shows the results obtained by using the new proposed method for the full response modulation case using the same parameters above except for $\omega_{c}=2 \pi 10^{6}, \nu=8$ and $M=8$ and the transmission of $10^{6}$ symbols. The demodulation was non-coherent, multiplying the received signal by $\cos \left(\omega_{c} t+\theta\right)$ and $\sin \left(\omega_{c} t+\theta\right)$ with $\theta$ being a random phase. The gain in performance comparing this result with the one shown in figure 2 is clearly seen. As already mentioned, this difference comes from the fact that the symbol can not be directly estimated in the first method.

This figure also compares two different scenarios: in the first one, named 'with LP filter', the receiver scheme is the one shown in figure 1. The low-pass (LP) filter used was a Butterworth filter with sufficient bandwidth not to distort the signal. In the simulated case, we used a bandwidth of $24.4 \mathrm{kHz}$. In the second scenario, named 'without LP filter', the received signal at the channel output $x$ is only multiplied by a cosine to give $x_{I}$ and by a sine to give $x_{Q}$. No low pass filter is used. Knowing that, in the process of recovering the symbols, it is necessary to calculate iterated integrals of this signals, this can be viewed as a low pass filtering and so the one in the receiver scheme would not be necessary. This second case render the receiver even simpler, without loss of performance (or even with a small gain), as can be seen in figure 3 .

Moreover, the difference between the performances of the receiver for CPFSK and LRC modulations is relatively small as expected since the similarity of their equations.

In addition, figure 4 shows the BER in the case of a bandlimited noise, for a CPFSK modulation with $h=0.15$ and $M=8$. The noise was obtained by first filtering a white noise with a baseband filter. This filter bandwidth is equal to the bandwidth occupied by the spectrum of the CPM signal, from its peak to a $30 \mathrm{~dB}$ attenuation. To obtain a noise centered at the same carrier frequency as the CPM signal, we proceed as follows [12]:

$$
\eta(t)=n_{I}(t) \cos \omega_{c} t-n_{Q}(t) \sin \omega_{c} t
$$

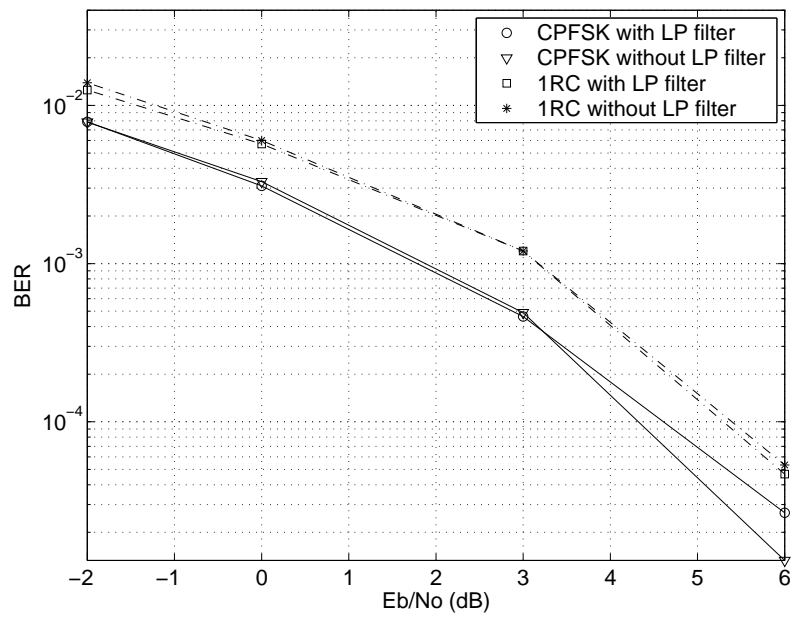

Fig. 3. New proposed method, full response modulation

where $n_{I}(t)$ and $n_{Q}(t)$ are independent noises obtained by filtering a white noise with a baseband filter. Figure 5 compares the baseband spectrum of the CPFSK signal with that of the colored noise. Both of them were normalized to start at $0 \mathrm{~dB}$. It is then possible to confirm that the noise spectrum is cut at a frequency equivalent to a $30 \mathrm{~dB}$ attenuation of the signal. Figure 6 shows a realization of $\eta(t)$, what confirms its time correlation.

Returning to the results shown in figure 4 , we observe that the method also has a good performance in this configuration. It is also important to note that the results are given as a function of the signal to noise ratio. Since we consider continuous time signals, the common approach (see e.g [12]) for simulating the noise corresponding to a given signal to noise ratio per bit is not adapted. Instead, we are considering bandlimited noise, a sample realization of which is displayed in figure 6. Figure 7 shows the noisy received signal for a SNR of $6 \mathrm{~dB}$.

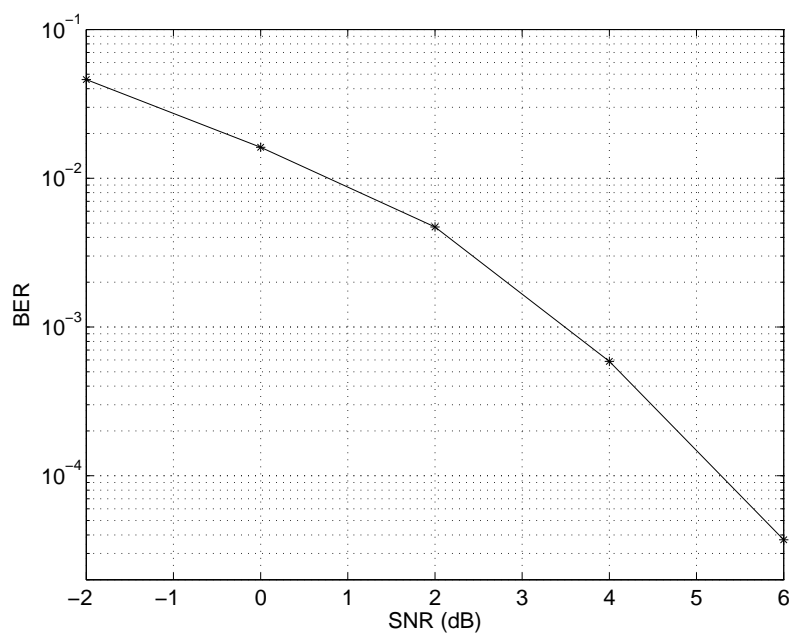

Fig. 4. Colored noise, CPFSK modulation 


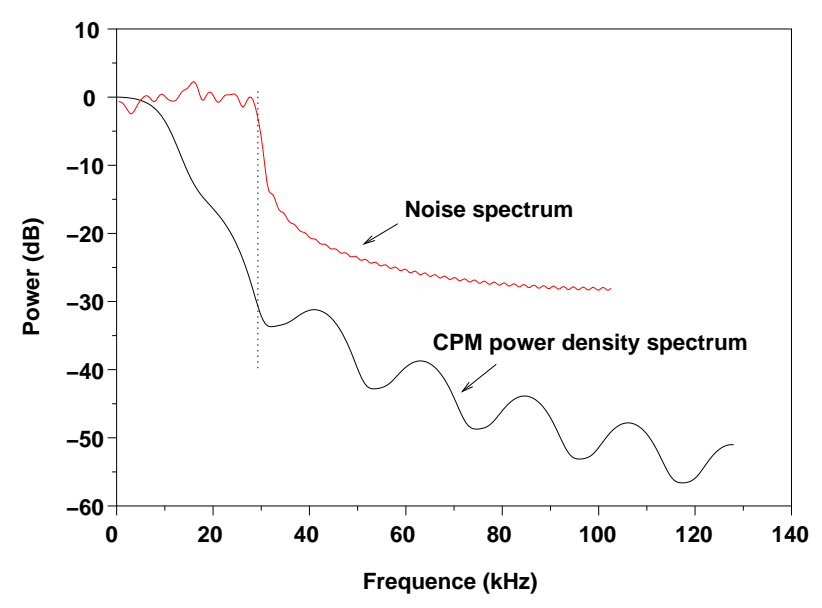

Fig. 5. CPM and colored noise spectrum

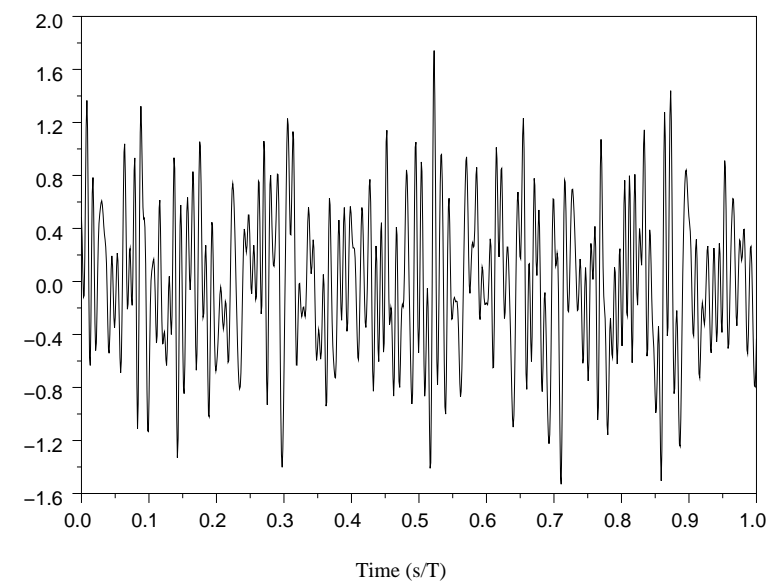

Fig. 6. Colored noise

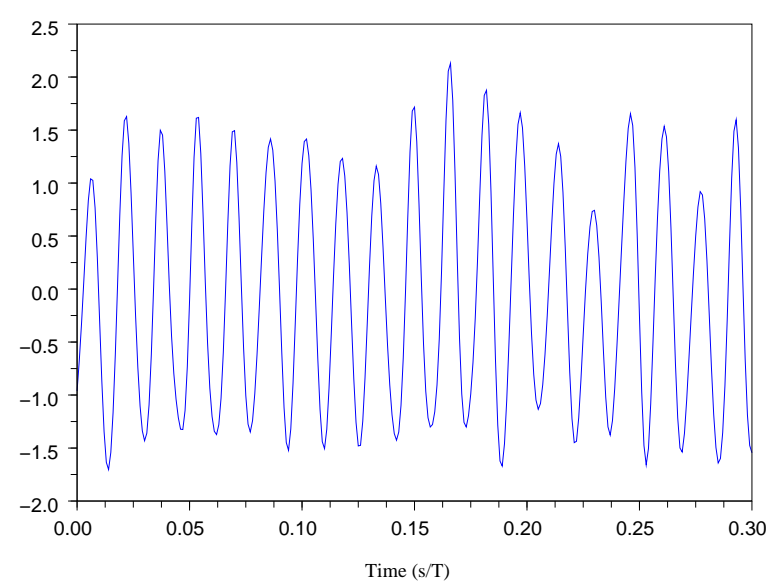

Fig. 7. Received signal, $\mathrm{SNR}=6 \mathrm{~dB}$

\section{CONCLUSION}

In this paper we proposed a new algebraic method for the demodulation of full response CPM signals in AWGN channel. The method consists on obtaining a closed formula for the symbols recovery that depends only on the received signal and on the knowledge of the modulation index. The proposed method is blind and very simple, being much more robust to noise than the one proposed firstly in [8]. It can also be equally used for coherent or non-coherent demodulation. We treated the CPFSK and the LRC families of CPM signals, showing that the resulting equations for both cases are very similar, as are their good performances.

This method can also be extended to other families of CPM signals and to the case of partial response modulations and selective channels, both of which are presently under study.

\section{ACKNOWLEDGMENTS}

This work was supported by FAPESP.

\section{REFERENCES}

[1] T. Aulin, N. Rydbeck, and C. Sundberg, "Continuous phase modulationPart I and II," IEEE Trans. on Comm., vol. 29, no. 3, pp. 196-225, 1981.

[2] J. Proakis, Digital Communications, 4th ed. Mc Graw-Hill, 2001.

[3] A. Svensson, C. Sundberg, and T. Aulin, "A class of reduced complexity viterbi detectors for partial response continuous phase modulation," IEEE Trans. on Comm., vol. 32, pp. 1079-1087, Oct. 1984.

[4] M. Gertsman and J. Lodge, "Symbol-by-symbol MAP demodulation of CPM and PSK signals on Rayleigh flat-fading channels," IEEE Trans. on Comm., vol. 45, pp. 788-799, July 1997.

[5] P. Bianchi, P. Loubaton, and F. Sirven, "On the blind equalization of continuous phase modulated signals using a constant modulus criterion," in Proc. IEEE Signal Processing Advances in Wireless Communications (SPAWC), Rome, 2003.

[6] _ "Blind joint estimation of the technical parameters of continuous phase modulated signals," in Proc. Globecom, 2003.

[7] G. Ford and S. Neugbauer, "Blind adaptive equalization in noncoherent receivers for continuous phase modulated signals," Project Report on MICRO Project 00-031, 2000-2001.

[8] M. Fliess, M. Mboup, and H. Mounier, "A new computational approach for the demodulation of CPM signals," in Proc. ISSPIT, Darmstadt, 2003.

[9] M. Fliess, M. Mboup, H. Mounier, and H. Sira-Ramírez, "Questioning some paradigms of signal processing via concrete examples," in Proc 1st Workshop Flatness, Signal Processing and State Estimate, CINVESTAVIPN, Mexico, D.F., 2003.

[10] M. Fliess and H. Sira-Ramírez, "An algebraic framework for linear identification," ESAIM Control Optim. Calculus Variations, no. 9, pp. 151-168, 2003.

[11] A. Neves, "Identification algébrique et déterministe de signaux et systèmes à temps continu: Application à des problèmes de communication numérique," Ph.D. dissertation, Université René Descartes - Paris $\mathrm{V}, 2005$.

[12] G. Wade, M. Fu, R. Jakobs, J. Ning, M. Tomlinson, and A. Ambroze, "On CPM system design and simulation," in Proc. IEEE Int. Conf. on Information Technology and Applications-Sydnei, Nov 2002. 